\title{
Gurltia paralysans: a neglected parasite of domestic cats
}

\author{
Marcelo Gómez ${ }^{\mathrm{a}}$, Manuel Moroni ${ }^{\mathrm{b}}$, Pamela Muñoz ${ }^{\mathrm{b}}$, Anja Taubert $^{\mathrm{c}}$, \\ Carlos Hermosillac ${ }^{c}$ Joerg Hirzmann ${ }^{c}$, Lisbeth Rojas ${ }^{\mathrm{a}}$
}

\begin{abstract}
Gurltia paralysans (order Strongylida; family Angiostrongylidae) is a metastrongyloid parasite that causes chronic meningomyelitis in domestic cats in South America. The geographic distribution of G. paralysans includes rural and peri-urban areas of Chile and Argentina. However, feline gurltiosis has recently been reported in other South American countries, including Uruguay, Colombia, and Brazil, and was also recently reported in Tenerife, Canary Islands (Spain). Feline gurltiosis is increasingly detected in domestic cats in southern Chile and its apparent geographic range is also increasing, together with an awareness of the disease among veterinarians. The life cycle of the parasite is unknown, but is probably indirect, involving gastropods as the intermediate host, as in other metastrongyloid nematode species. The clinical signs of $G$. paralysans infection include progressive pelvic limb ataxia, paraparesis, paraplegia, faecal or urinary incontinence, and/or tail paralysis. A definitive diagnosis of feline gurltiosis is still challenging and only possible with necropsy, when adult G. paralysans nematodes are detected within the spinal cord vasculature, together with macroscopic lesions, and characteristic morphological features. A semi-nested PCR method was recently developed for the in vivo diagnosis of this neglected parasite. Current treatment options include macrocyclic lactones and mylbemicn oxime, but the prognosis is poor in severe cases. In this article, we review G. paralysans infection in cats, focusing on the diagnosis shortcomings and the future directions of research into its biology and the associated neurological disease. Comprehensive updates on the epidemiology and clinical features, diagnosis, treatment, and prevention of feline gurltiosis are provided.

Key words: Gurltia, feline, nematode, spinal cord.
\end{abstract}

\section{INTRODUCTION}

Gurltia paralysans (Nematoda; order Strongylida; superfamily Metastrongyloidea; family Angiostrongylidae) is a metastrongyloid parasite that causes chronic meningomyelitis in domestic cats (Wolffhügel 1933, Levin 1968, Bowman et al 2002, Gómez et al 2010, Moroni et al 2012). The geographic distribution of G. paralysans includes areas of Chile, Argentina, Uruguay, Colombia, and Brazil (Guerrero et al 2011, Gómez-Alzate et al 2011, Rivero et al 2011, Moroni et al 2012, Melo Neto et al 2019) (figure 1), but recently has been isolated in the Canary Islands, Spain (Udiz-Rodríguez et al 2018). The nematode can be found in the leptomeningeal veins and the parenchyma of the spinal cord of the feline host, and the infection has been associated to progressive paraparesis, paraplegia, faecal or urinary incontinence, and/or tail paralysis (Gómez et al 2010, Moroni et al 2012, Mieres et al 2013). The life cycle of the parasite is unknown, but is likely to be heteroxenous, as other metastrongyloid nematodes. The diagnosis of feline gurltiosis is challenging and, so far, only possible by necropsy (Wolffhügel 1933, Wolffhügel 1934, Moroni et al 2012, Muñoz et al 2017).

Received: 15.11 .2019

Accepted: 14.05.2020

anstituto de Farmacología y Morfofisiología, Facultad de Ciencias Veterinarias, Universidad Austral de Chile, Valdivia, Chile.

bInstituto de Patología Animal, Facultad de Ciencias Veterinarias, Universidad Austral de Chile, Valdivia, Chile.

'Institute of Parasitology, Justus Liebig University Giessen, Giessen, Germany.

*Corresponding author: M Gómez; marcelogomez@uach.cl

\section{HISTORICAL PERSPECTIVE}

Gurltia paralysans was first reported in the early 1930s in Chile by Kurt Wolfgang Wolffhügel (1869-1951), a German scientist, naturalist, and parasitologist (Wolffhügel 1934, Bowman et al 2002). Wolffhügel diagnosed eleven cases of domestic cats, studying their pathological lesions and extracting adult nematodes from their spinal cords. All affected animals came from the Provinces of Llanquihue and Puerto Varas, Southern Chile (latitude $41^{\circ}$ south). The genus Gurltia was named after Ernst Friedrich Gurlt (17941882), a German veterinary anatomist and teratologist. Wolffhügel called the disease "paraplejia cruralis parasitaria felis" and initially placed the nematode as genus Hemostrongylus, later called Angiostrongylus.

\section{MORPHOLOGICAL CHARACTERISTICS}

The measurements of male and female specimens reported in the literature are summarised in table 1. Males of G. paralysans have a body length of 12-18 $\mathrm{mm}$ and are $0.072 \mathrm{~mm}$ wide just anterior to the bursa, with a $0.026-0.032 \mathrm{~mm}$ wide in the cephalic region (Wolffhügel 1934, Moroni et al 2012, Rodríguez 2013, Muñoz et al 2017). The oesophagus is 0.368-0.0392 $\mathrm{mm}$ long, and the oesophago-intestinal junction is 0.008 $\mathrm{mm}$ long. The nerve ring is $0.148-0.164 \mathrm{~mm}$ in diameter and the excretory pore occurs $0.232-0.240 \mathrm{~mm}$ from the anterior end (Muñoz et al 2017) (figure 2, table 1). No cervical papillae are reported. The spicules are 0.65$0.902 \mathrm{~mm}$ long, curved in lateral view, and the tip has a main stem and a single-pointed branch surrounded by a bluntly rounded membrane (Wolffhügel 1934, Moroni 
Table 1. Measurements of morphological characters of male and female Gurltia paralysans reported in the literature.

\begin{tabular}{|c|c|c|c|c|c|c|c|c|}
\hline \multirow[t]{2}{*}{ Characters } & \multicolumn{4}{|c|}{ Male } & \multicolumn{4}{|c|}{ Female } \\
\hline & $\begin{array}{l}\text { Wolffhügel } \\
1934\end{array}$ & $\begin{array}{c}\text { Moroni et al } \\
2012 \\
(\mathrm{n}=4)\end{array}$ & $\begin{array}{c}\text { Rodríguez } \\
2013 \\
(\mathrm{n}=7)\end{array}$ & $\begin{array}{c}\text { Muñoz et al } \\
2017 \\
(\mathrm{n}=7)\end{array}$ & $\begin{array}{c}\text { Wolffhügel } \\
1934\end{array}$ & $\begin{array}{c}\text { Moroni et al } \\
2012 \\
(\mathrm{n}=6)\end{array}$ & $\begin{array}{c}\text { Rodríguez } \\
2013 \\
(\mathrm{n}=14)\end{array}$ & $\begin{array}{c}\text { Muñoz et al } \\
2017 \\
(\mathrm{n}=2)\end{array}$ \\
\hline Total length & 12 & $13-18$ & $10.20-16.35$ & $14-15$ & $20.5-23.0$ & $23-30$ & $23.55-36.06$ & $27-28$ \\
\hline $\begin{array}{l}\text { Maximum } \\
\text { width }\end{array}$ & 0.07 & 0.1 & $0.075-0.078$ & 0.072 & - & 0.1 & $0.113-0.150$ & $0.082-0.088$ \\
\hline $\begin{array}{l}\text { Oesophagus } \\
\text { length }\end{array}$ & $0.360-0.432$ & 0.4 & $0.225-0.338$ & $0.036-0.039$ & - & 0.6 & $0.226-0.338$ & $0.444-0.468$ \\
\hline Nerve ring & - & - & - & $0.148-0.164$ & - & - & - & 0.132 \\
\hline $\begin{array}{l}\text { Nerve ring/ } \\
\text { anterior end } \\
\text { distance }\end{array}$ & - & - & - & $0.075-0.114$ & - & - & - & - \\
\hline Excretory pore & - & - & - & $0.232-0.240$ & & & & 0.236 \\
\hline $\begin{array}{l}\text { Anal/end } \\
\text { distance }\end{array}$ & - & - & - & - & - & 0.03 & $0.001-0.038$ & - \\
\hline $\begin{array}{l}\text { Anal/vulva } \\
\text { distance }\end{array}$ & - & - & - & - & - & 0.13 & - & - \\
\hline $\begin{array}{l}\text { Vulva/end } \\
\text { distance }\end{array}$ & - & - & - & - & 0.150 & - & $0.112-0.171$ & $0.102-0.112$ \\
\hline Spicules length & 0.65 & 0.8 & $0.756-0.902$ & $0.722-0.816$ & - & - & - & - \\
\hline Spicule width & - & 0.003 & - & - & - & - & - & - \\
\hline $\begin{array}{l}\text { Gubernaculum } \\
\text { length }\end{array}$ & - & - & $0.037-0.039$ & 0.062 & - & - & - & - \\
\hline Bursa width & - & 0.13 & $0.076-0.150$ & - & - & - & - & - \\
\hline Bursa length & - & 0.066 & $0.038-0.039$ & - & - & - & - & - \\
\hline Egg length & - & - & - & - & $0.05-0.065$ & - & - & $0.040-0.72$ \\
\hline Egg width & & & & & $0.039-0.054$ & & & $0.026-0.048$ \\
\hline
\end{tabular}

All measurements are given in millimetres $(\mathrm{mm})$.

et al 2012, Rodríguez 2013, Muñoz et al 2017). The male gubernaculum is $0.062 \mathrm{~mm}$ long, slender, curved, and tapering distally in lateral view (Moroni et al 2012, Rodríguez 2013, Muñoz et al 2017). The bursal lobes are symmetrical and the bursal rays are arranged with the ventral rays, rays 2 and 3, fused to a common stem and only separated distally. The lateral rays have a common stem and the anterolateral ray (ray 4) branches off first and is longer than the other 2 laterals (rays 5 and 6), which share a common stem. The external dorsal ray (ray 8) is shorter than the lateral rays and appears segmented in mature specimens; the dorsal rays (rays 9 and 10) have thick stems, with some variation in length between the 2 male specimens examined, and only divide into 2 small branches at the distal tip. The genital cone has a ventral cone-shaped membrane (Muñoz et al 2017). Females have a body length of 20.5-36.06 $\mathrm{mm}$ and a width of
0.082-0.088 $\mathrm{mm}$ just anterior to the vulva (Wolffhügel 1934, Moroni et al 2012, Rodríguez 2013, Muñoz et al 2017). The cephalic region is $0.032-0.036 \mathrm{~mm}$ wide. The oesophagus is $0.444-0.468 \mathrm{~mm}$ long and the oesophago-intestinal valve is $0.008-0.012 \mathrm{~mm}$ in diameter. The nerve ring is $0.132 \mathrm{~mm}$ in diameter and the excretory pore occurs $0.236 \mathrm{~mm}$ from the anterior end. The vulva opens $0.102-0.112 \mathrm{~mm}$ from the tail tip, and the posterior margin of the vulva has a folded flap in mature specimens (figure 2). No didelphic or prodelphic infundibula or sphincters are visible. The uteri join to form a vagina $1.44 \mathrm{~mm}$ from the vulval opening. The eggs in the uterus and vagina are $0.040-072 \mathrm{~mm} \times 0.026-0.048 \mathrm{~mm}$ in size, depending on maturity. Scanning electron microscopic images have revealed the presence of double submedian cephalic papillae, amphids, and a lip with a tooth at the anterior end of the parasite (Muñoz et al 2017). 


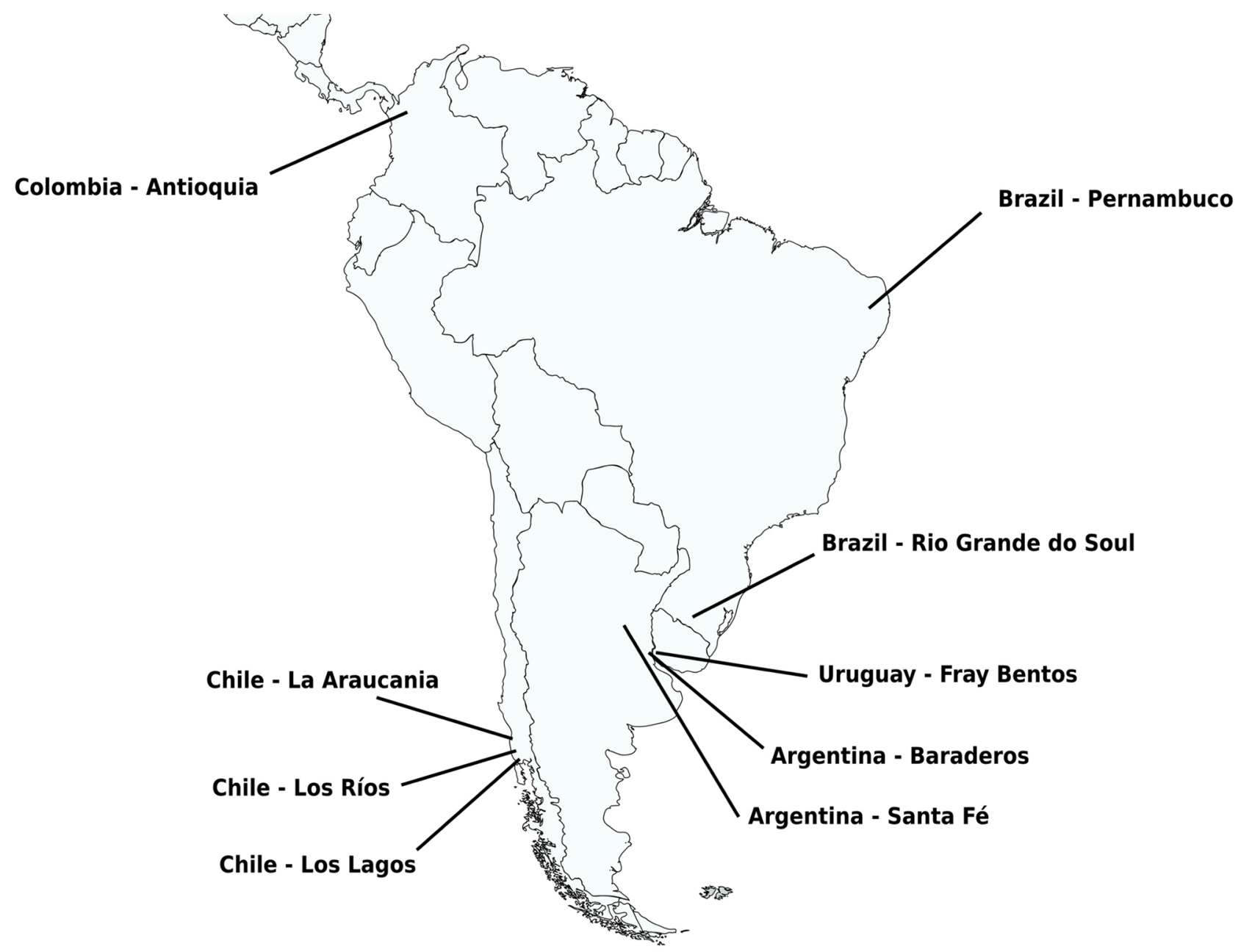

Figure 1. Geographic localisation of reports of natural infections of Gurltia paralysans in South America.

\section{LIFE CYCLE OF G. PARALYSANS}

The transmission or life cycle of G. paralysans is mostly unknown. Adult males and females and eggs have been found in the veins from the subarachnoid space of the host spinal cord (Moroni et al 2012). No eggs or larvae were found in the faeces, blood, bronchial lavage, or other body fluids of eight infected cats (Peña 2014). As other closely related metastrongyloid species infecting felines, terrestrial gastropods as intermediate hosts (IH) and/or paratenic hosts $(\mathrm{PH})$ have been hypothesised in the life cycle of G. paralysans. Thus, cats may become infected by consuming G. paralysans-carrying gastropods (i.e., slugs and snails) or a $\mathrm{PH}$ with an infective third-stage larvae (L3), including insects, frogs, toads, lizards, birds, and rodents (Moroni et al 2012, Melo Neto et al 2019) (figure 3). In a recent study, 835 terrestrial gastropods, including members of the Fam. Arionidae, Limacidae, Helicidae, and Milacida, were collected during August 2015 and November 2016 in Valdivia, Southern Chile, close to reported cases of gurltiosis (Sepúlveda 2018).
All gastropods were subjected to enzymatic digestion to isolate G. paralysans larvae. Ten per cent of the gastropod samples were analysed with semi-nested PCR targeting the $18 \mathrm{~S}$ ribosomal RNA (rRNA) gene, and $2.6 \%$ were analysed with histopathology (Sepúlveda 2018). However, the results of the study indicated the absence of G. paralysans by the three methods used. Thus, the molluscan species analysed may not act as $\mathrm{IH}$, and further studies are required to evaluate the role of other species of aquatic gastropods in this geographic area (Sepúlveda 2018).

In a hypothetical migration route, a mollusc or $\mathrm{PH}$ is ingested by a cat. The infective larvae (L3) migrate via the mucosae of the digestive system to the veins or lymphatic system of the abdominal viscera and then via connections of the azygos or caval venous system with thoracic, lumbar or sacral intervertebral veins to reach the vertebral venous plexus (VVP) (figure 3 ). The VVP is in direct communication with the cranial venous system, and because no valves exist in either of them, the blood may flow cranially or caudally, depending on the pressure relationship (Gómez \& Freeman 2004). The valveless VVP 


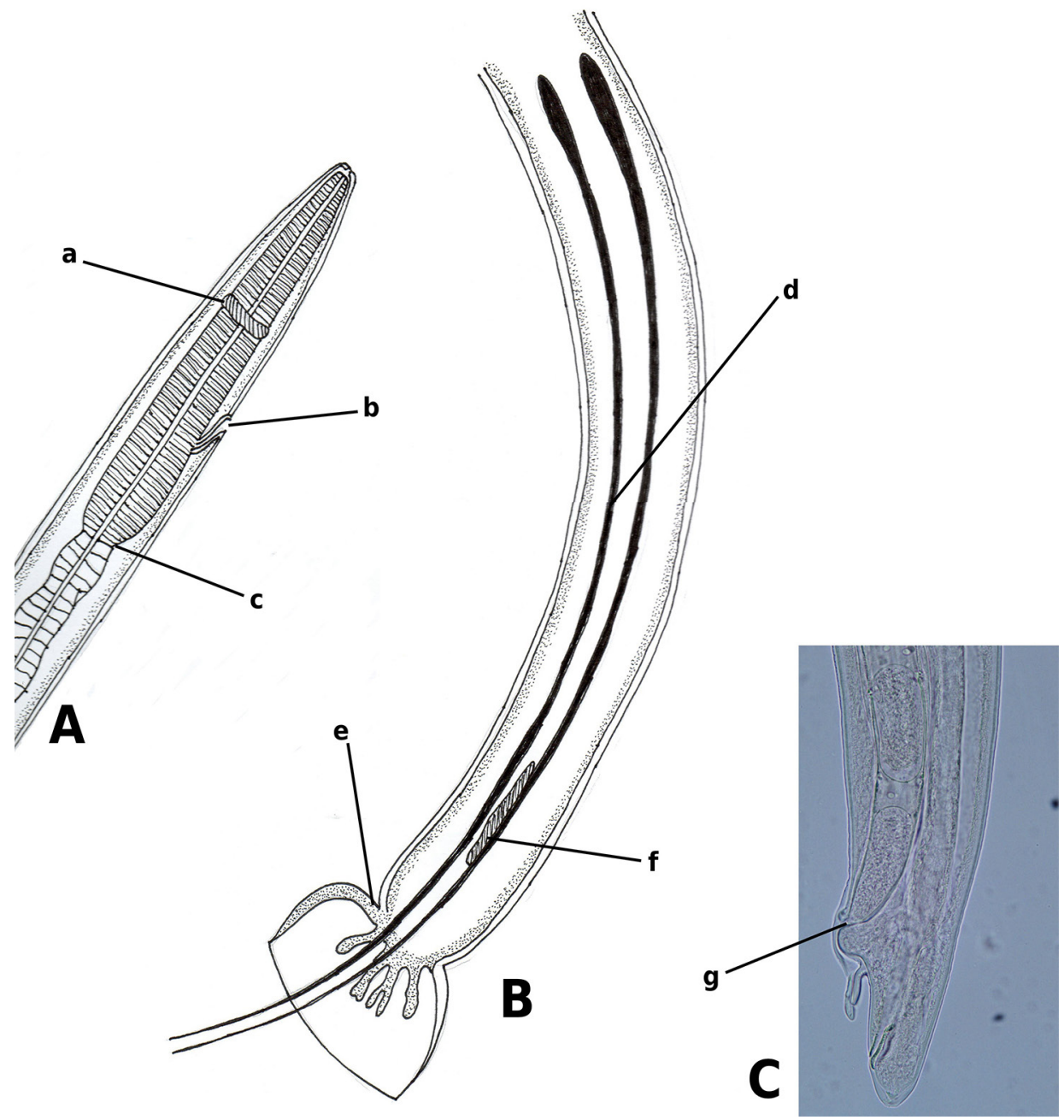

Figure 2. Schematic morphology of Gurltia paralysans. Adult (A), cranial end. Note the nerve ring (a), anal (excretory) pore (b), and oesophago-intestinal junction (c). Male adult (B), caudal end, showing the spicules (d), bursal rays (e), and gubernaculum (f). Photograph of a female adult larva (C), caudal end, showing the vulva and anus (g). (Illustration and photograph by Marcelo Gómez).

is probably used by G. paralysans to reach the spinal subarachnoid space or even the brain (Katchanov and Nawa 2010, Moroni et al 2012). In spinal schistosomiasis in humans, the dissemination of the parasite occurs via the intestinal veins to the VVP (Shahlaie et al 2005). Spinal schistosomiasis usually involves the lower thoracic and lumbosacral spine, probably because the VVP connects the intra-abdominal veins with those of the lower spine (Paz et al 2002). Consistent with this, the presence of adult specimens or eggs of $G$. paralysans within the ventral VVP (video 1$)^{1}$ and the basivertebral veins located in the vertebral bodies (figure 4) has been observed (unpublished data). These venous connections could also explain the presence of eggs and adult $G$. paralysans in distant places, such as the cerebrum and the anterior chamber of the eye,

$1 \quad$ Available at www.australjvs.cl/ajvs in more recent reports (Figueroa 2017, Udiz-Rodríguez et al 2018, Melo Neto et al 2019). The adaptation of this nematode to migrate exclusively into the venous system may be associated with abiotic factors in the vein environment, such as chemical $\left(\mathrm{CO}_{2}\right.$ concentration, $\mathrm{O}_{2}$ concentration $)$ and physical factors (temperature, mechanics), nutrients, etc (Read \& Sharping 1995). Critical gaps for future research in the migratory pathway of G. paralysans include the vein tropism of adults and the neuroanatomical localisation to the subarachnoid veins of the spinal cord.

To date, all reports of feline gurltiosis have shown that domestic cats are predominantly affected. However, recent reports from Brazil have described the isolation of G. paralysans from the lumbar spinal cord segments of adult wild cats, including the northern tiger cat (Leopardus triginus) and a female margay (L. wieddi) (Oliveira 2015, Dazzi et al 2020). Although the huiña or kodkod (L. guigna) and Geoffroy's cat (L. geoffroyi) have been 


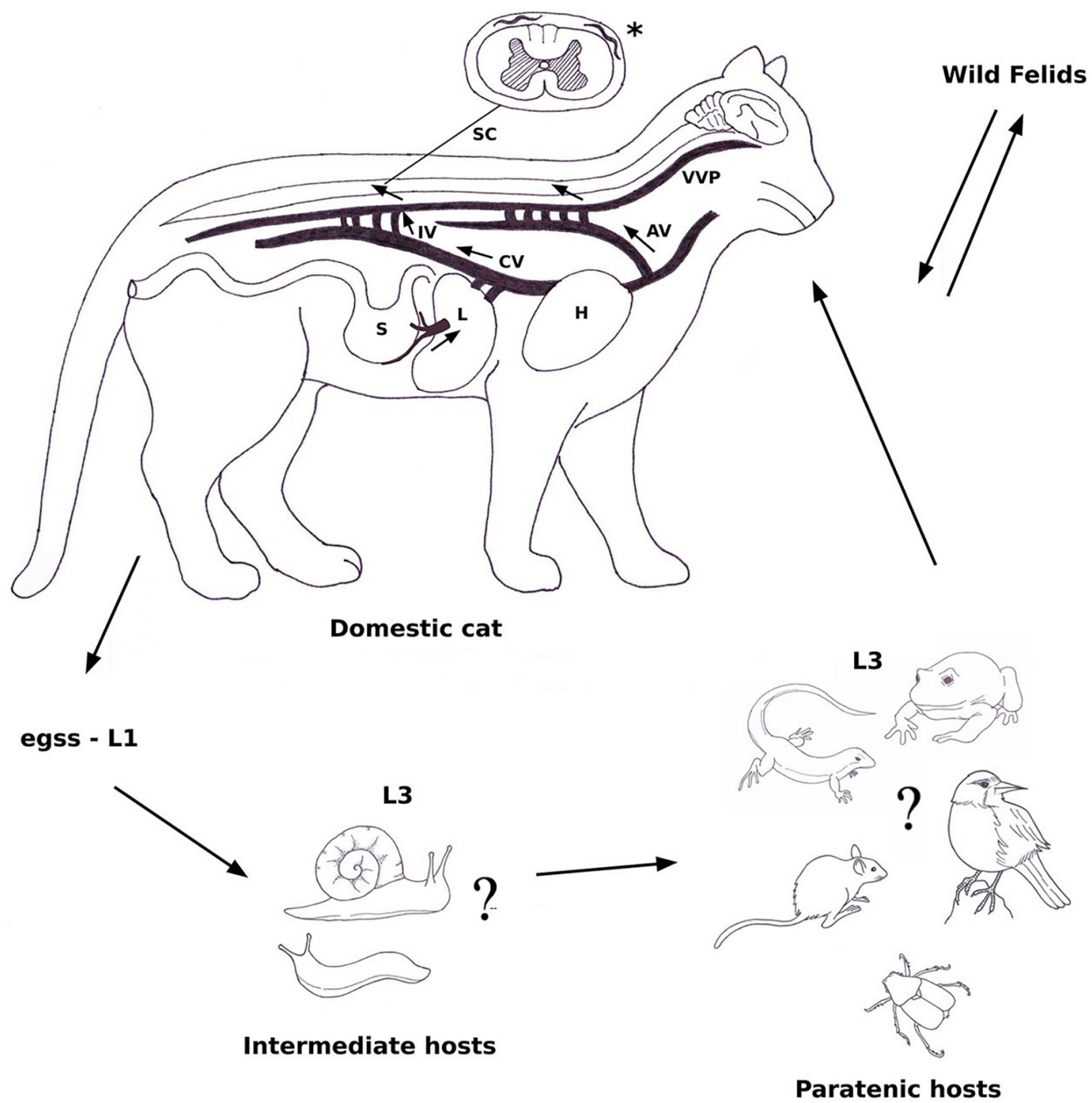

Figure 3. Proposed life cycle and migration pathways of Gurltia paralysans. Domestic cats or wild felids acquire the L3 larvae by ingesting an infected intermediate host (gastropods) or paratenic host (lizards, rodents, amphibians, birds, or insects). Infective larvae penetrate the stomach and enter the hepatic portal system and then the caudal vena cava and/or the azygous venous system. From those vein systems, the larvae migrate to the spinal cord via the intervertebral veins and the vertebral venous plexus. The larvae invade the veins of the subarachnoid space of the spinal cord, where they mature (asterisk) and lay eggs. It is still unknown how domestic cats eliminate the eggs or first larval stage (L1), or how the intermediate host becomes infected with L1. AV: azygos vein; CV: caudal vena cava; IV: intervertebral veins; H: heart; L: liver; S: stomach; SC: spinal cord; VVP: vertebral venous plexus; L1: first-stage larvae; L3: third-stage larvae. (Illustration by Marcelo Gómez).

suggested as possible definitive hosts, neither larval stages nor clinical cases have been diagnosed yet in these wild felids (Muñoz et al 2017). Further molecular studies are necessary to determine the role of wild cats as hosts and the transmission to other species.

\section{EPIDEMIOLOGY}

The first cases of feline gurltiosis were reported in domestic cats from areas of the Valdivian rainforest in southern Chile (Wolffhügel 1933). In Chile, cases has been reported in the La Araucanía, Los Ríos, and Los Lagos regions (Gómez et al 2010, Moroni et al 2012). Since then, cases have been reported in Argentina (Guerrero et al 2011, Bono et al 2016), Uruguay (Rivero et al 2011), Colombia (Gómez et al 2011), and Brazil (Togni et al 2013, Melo Neto et al 2019) (figure 1). In Chile, cases of feline gurltiosis have predominantly been diagnosed in rural areas, but more specific spatial distribution in this environment is poorly known (Wolffhügel 1933, Gómez 

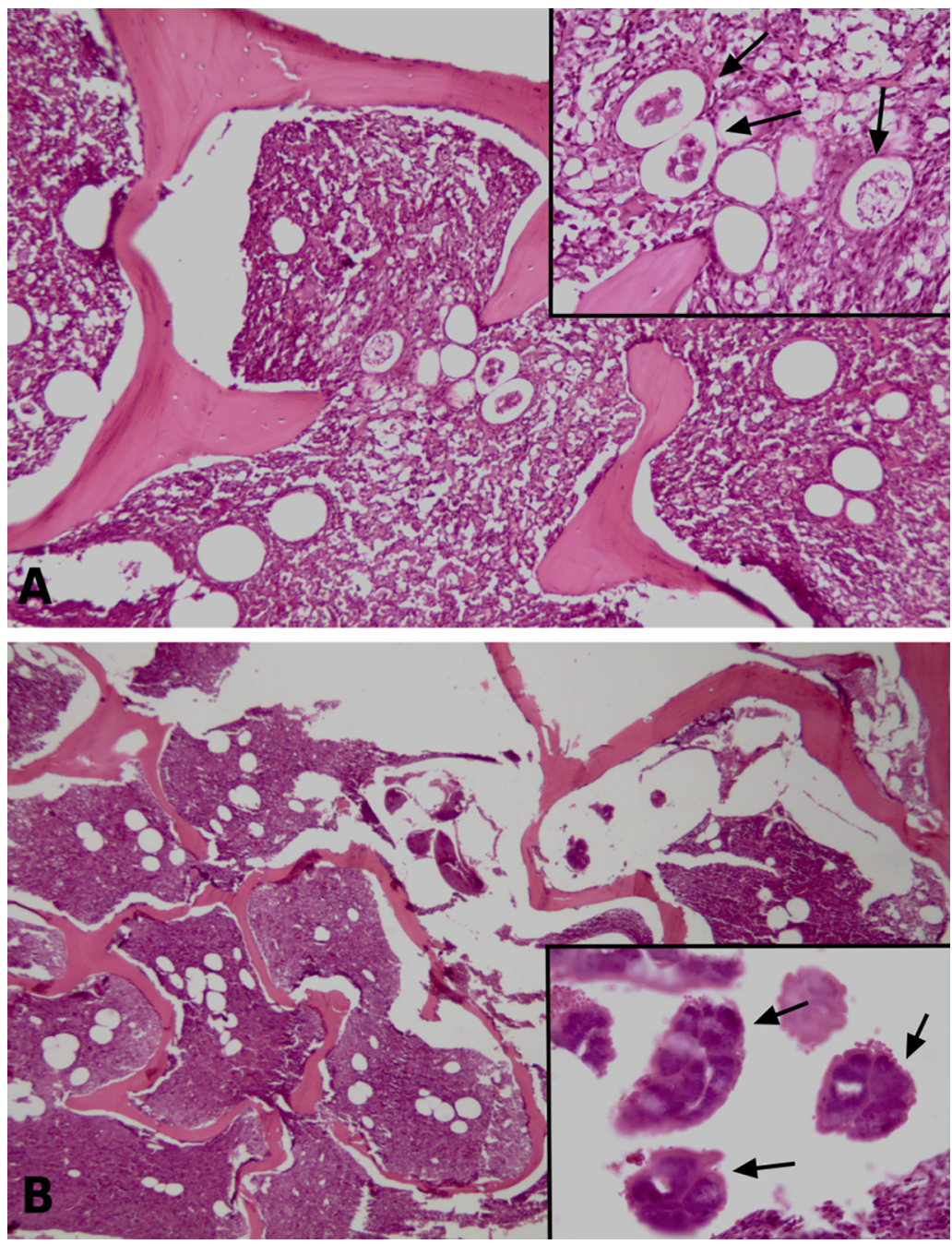

Figure 4. Presence of embryonated eggs (cross-section) (arrows) (A) and fragments of an adult specimen (arrows) (B) of Gurltia paralysans in the spongiosa of a lumbar vertebral body from an affected domestic cat. Inserts show augmented views $(40 \times)$.

et al 2010, Moroni et al 2012, Mieres et al 2013). The disease has been diagnosed in three different regions in the southern part of Chile: the Araucanía region (e.g., Lastarria), the Los Rios region (e.g., Punucapa, Niebla, Paillaco, and Futrono), and the Los Lagos region (e.g., Futrono, Pichirropulli, and Ancud) (Moroni et al 2012). In Colombia, six cases of gurltiosis in domestic cats were reported in Antioquia (municipalities Tarso and Amagá) with signs of pelvic limb weakness and spinal hyperesthesia (Alzate et al 2011). In Argentina, in 2011, one cat with $G$. paralysans was reported in the Baradero area of the province of Buenos Aires and three cases in rural areas of the Santa Fé province, in the districts of Las Colonias, San Cristobal, and Castellanos (Guerrero et al 2011, Bono et al 2016). In Uruguay, two cases of feline parasitic meningomyelitis due to G. paralysans were described between 2008 and 2009 in the rural area of Fray Bentos (Rivero et al 2011). This parasitosis has been recognised in Brazil since the mid-1990s, under the local name "bambeira", "derrengado", or "renga" (Togni et al 2013). In 2013, four cats with G. paralysans infections were reported in the state of Rio Grande do Sul (Togni et al 2013), and eleven cases were recently found in the state of Pernambuco (municipalities of Caetés and Capoeiras), northeast Brazil (Melo Neto et al 2019). Also in Brazil, two wild cats (L. wieddii and L. triginus) were found in the state of Parana (Chapecó) with classical post-mortem spinal cord lesions of feline gurltiosis (Oliveira 2015, Dazzi et al 2020). More recently, the first case outside of America was reported in Tenerife (Canary Islands, Spain) (Udiz-Rodriguez et al 2018). In 1993, a cat with neurological and necropsy findings in the lumbar spine compatible with feline gurltiosis was reported in the United States (Bowman et al 2002). Both cases of G. paralysans, in the Canary Islands and in the US, may have resulted from the introduction of $G$. paralysans-infected domestic cats from endemic areas of South America or the importation of infected IH, as 
reported for Angiostrongylus cantonensis introduced to Canary island (Foronda et al 2010), although further research is still required.

The disease has been associated with rural settings and rainforest areas, with humid ecosystems and abundant vegetation (Wolffhügel 1933, Gómez et al 2009, Rivero et al 2011, Togni et al 2013, Melo Neto et al 2019). The prevalence of the nematode in South America is unknown, but is likely to be underestimated and the disease under-diagnosed (Muñoz et al 2017). Modelling studies have indicated that southern Chile and Argentina, and the areas in Brazil, Uruguay, and Colombia, where gurltiosis has been diagnosed, are regions at clear risk of the spread of angiostrongyloid nematodes based on their climatic suitability (Morgan et al 2009). No seasonal occurrence pattern has yet been reported for feline gurltiosis, which is detected in all seasons of the year (Gómez et al 2010, Moroni et al 2011, Rivero et al 2011). In one report, cats with gurltiosis were all co-infected with Aelurostrongylus abstrusus, despite none of them showing respiratory symptoms (Mieres et al 2013). This could indicate that cats infected with $G$. paralysans and A. abstrusus could share IH or PH. Factors affecting the distribution of gastropod species are important in determining whether the life cycle of $G$. paralysans can be completed and the potential contact with suitable hosts. Previous studies have indicated that the range of metastrongyloid parasites (i.e., Angiostrongylus vasorum) has expanded into new countries and regions (Traversa et al 2010, Maksimov et al 2017, Lange et al 2018). Models have been used to predict the distribution of $A$. vasorum and the risk of infection based on climatic variables and their effects on the survival rates of the intermediate hosts (Maksimov et al 2017). Similar modeling information is required for G. paralysans to predict the distribution range. The causes of the apparent re-emergence of metastrongyloid parasitoses in domestic animals are still unknown, but several factors may explain the recent increases in reports of feline gurltiosis in several countries (Traversa et al 2010, Melo Neto et al 2019). These include global warming, changes in the seasonal population dynamics of vectors, and massive movement of animals (Traversa et al 2010, Maksimov et al 2017). Further investigations on this neglected field are needed, including identifying the exposure by serological $/ \mathrm{molec}$ ular prevalence investigations, specific local and global geographic ranges of feline gurltiosis and determining the epidemiological and climatic factors that allow the establishment of G. paralysans infection.

\section{PATHOLOGICAL LESIONS}

The necropsy findings in infected domestic cats include diffuse submeningeal congestion of the lumbar, sacral, and coccygeal spinal cord segments (Gómez et al 2010, Moroni et al 2012). Several intravascular nematode larvae and pre-adult stages can be identified histologically in the meningeal veins of the spinal cord, associated with congestion, thrombosis, and thickening of the subarachnoidal vessels (Gómez et al 2010, Moroni et al 2012, Mieres et al 2013, Togni et al 2013, Moroni et al 2017). Studies have reported mild smooth-muscle hypertrophy, moderate adventitial fibroplasia, and marked subintimal fibrosis of the spinal cord venules (phlebosclerosis) (Togni et al 2013). In some specimens, concentric thickening of the venule wall may produce stenosis of the vessel lumen (Togni et al 2013). Intraluminal papillary projections with an arboriform appearance to the interior of the dilated venules have been interpreted as varicose lesions (venular varices) (Moroni et al 2012, Togni et al 2013). Sections of normal or dilated and tortuous varicose venules may contain thrombi with various levels of organisation (Togni et al 2013). The spinal cord parenchyma may show multiple haemorrhages and extensive foci of malacia, with gitter cells and adjacent gliosis (Togni et al 2013). Lymphocytes, intermingled with fewer macrophages, primarily infiltrate the meninges, forming a perivascular pattern. Mature eosinophils scattered randomly within the leptomeninges have also been observed, which are consistent with extensive spinal leptomeningitis and thrombophlebitis (Moroni et al 2012, Togni et al 2013). Some animals may also show granulomatous leptomeningitis or suppurative leptomeningitis (Togni et al 2013). White-matter lesions in the spinal cord segments may have variable degrees of Wallerian degeneration, characterised by the distension of the myelin sheath diameter, irregular axons, axonal swelling, bulbous axonal fragmentation (caused by the presence of axonal spheroids), microcavitation, and focal areas of mineralisation (Moroni et al 2012, Togni et al 2013, Moroni et al 2017). Varicose venules can also be observed in the white matter of the spinal cord, but are associated with recesses in the meninges (Togni et al 2013). The activation of glial and endothelial cells and immune cell infiltration, visualised with immunohistochemical markers (i.e. GFAP, CNPase, factor VIII, CD3, and $\mathrm{CD} 45 \mathrm{R}$ ) in affected spinal cord samples, indicate gliosis and chronic inflammatory spinal cord lesions subsequent to the ischemia caused by parasitic vascular injury (Vienenkotter et al 2015, Jara 2018). The predominant cellular infiltrate in the affected spinal cord is of the mononuclear type, indicating the chronic nature of the lesions (Jara 2018). A recent study analysed the presence of histopathological lesions in the cerebrum, cerebellum, and brainstem in 13 feline patients with post-mortem spinal lesions due to G. paralysans (Figueroa 2017). Congestion and hyperaemia were observed in the peripheral blood vessels of both the dorsal and ventral zones of the cerebrum in the 13 cats. In 7 cases, mononuclear cell infiltrate was observed around the choroid plexus, the third and fourth ventricles, and associated blood vessels. Six cats showed thickening of the meninges and 2 showed perivascular neutrophilic inflammatory infiltrate at the level of the cerebral subarachnoid space (Figueroa 2017). Similar findings of leptomeningeal vascular congestion, varices, 
and perivascular cellular infiltrate were observed in the encephalons (frontal, temporal, and occipital cortices) of 11 cats infected with G. paralysans in Brazil (Melo Neto et al 2019). However, no clinical cases of feline gurltiosis have been observed with clinical cerebral, cerebellar, or brainstem syndromes.

Histological samples from ten $G$. paralysans-infected cats were analysed at the hepatic level. All samples showed signs of periportal hepatic degeneration, periportal inflammatory infiltration, comprising neutrophilic and mononuclear infiltration, indicating direct injury to the liver (Verscheure 2014). However, the possible mechanisms by which intravascular parasites could cause this type of injury pattern in the liver remains unclear. Angiostrongylus vasorum can induce moderate liver parenchymal parasitic hepatitis and lesions such as interstitial hemorrhage disseminated inflammatory cells in the portobiliar space or around centrolobular veins attributable to larval nematode migration (Rinaldi et al 2014, Cook et al 2015). In kidneys, gurltiosis has been associated to the presence of hyaline protein deposits inside Bowman's capsule (in 8 out of 10 cases), the thickening of Bowman's capsule in five cases, and the presence of interstitial inflammatory infiltrate, consisting of neutrophils and eosinophils (in 4 out of 10 cases) (Verscheure 2014). These findings are compatible with glomerulonephritis, which could have been immune-mediated by the deposition of immune complexes of the host (Verscheure 2014).

Further studies are required to understand the role of the immune pathogenesis of the disease, such as interleukins, cytokines, and host cells (e.g., eosinophils, neutrophils, plasma cells, T cells) and how $G$. paralysans regulates feline endothelial functions (e.g. via excretory or secretory antigens) allowing the parasite to use intravascular habitat.

\section{CLINICAL SIGNS}

The most common clinical manifestation of feline gurltiosis is chronic and progressive ambulatory paraplegia (Gómez et al 2010, Gómez et al 2011, Rivero et al 2011, Mieres et al 2013, Togni et al 2013). Based on 19 cases, the duration of the clinical signs ranges from 2 weeks to 48 months (Mieres et al 2013, Rivero et al 2011, Togni et al 2013, Bono et al 2016, Moroni et al 2016). Other clinical signs include pelvic limb ataxia, pelvic limb proprioceptive deficit, pelvic limb tremor, pelvic limb muscle atrophy, tail trembling, tail atony, and faecal and urinary incontinence (Gómez et al 2010, 2011, Gómez et al 2011, Guerrero et al 2011, Rivero et al 2011, Moroni et al 2012, Mieres et al 2013, Togni et al 2013, MeloNeto et al 2019). The neurological signs are associated with the neuroanatomical lesions observed at necropsy and in histopathological specimens (Mieres et al 2013). The associated haematological abnormalities included non-regenerative anaemia and low mean corpuscular haemoglobin concentrations (hypochromia) (Rojas 2011, Mieres et al
2013) indicating chronic inflammatory disease or chronic blood loss (Gredal 2011). The eosinophilia associated with parasitism has commonly been reported in domestic animals, but is not a common finding in cats with gurltiosis (Mieres et al 2013), which has also been reported in dogs with neural angiostrongylosis (Bourque 2002). No signs of coagulopathy have been observed in naturally infected cats. However, high levels of urea in the blood have been reported, probably arising from neurogenic urinary dysfunction (Mieres et al 2013). A bronchial lavage analysis of five naturally $G$. paralysans-infected cats, showed the absence of larval stages and eggs (Peña 2014). Ocular lesions (uveitis, chorioretinitis, posterior synechiae, corneal oedema) have recently been reported to be associated with the presence of a motile adult specimen of $G$. paralysans in the anterior chamber of the eye in a domestic cat (Udiz-Rodríguez et al 2018).

\section{DIAGNOSIS}

Imaging studies by computed tomography, myelography, and magnetic resonance imaging (MRI), indicate that $G$. paralysans induce lesions in the thoracolumbar, lumbar, and sacral regions, suggesting diffuse inflammatory spinal cord lesions (Gómez et al 2010, Mieres et al 2013, Togni et al 2013). Myelograms show the retention of columns of contrast medium in the thoracolumbar region (Mieres et al 2013). The intramedullary accumulation of contrast medium, similar to the pattern for myelomalacia, in the thoracolumbar spinal cord segment has been reported in infected cats (Guerrero et al 2011), similar to myelographic examinations of dogs with A. vasorum infections (Lun et al 2012). However, the myelographic evaluations in dogs with intramedullary parasitic infections attributed to Spirocerca lupi are normal (Chai et al 2008). MRI images of affected cats show multiple nodular areas of hyperintensity in the periphery of the spinal cord, which could also represent slow venous flow within the perimedullary veins (Mieres et al 2013) (figure 5). Spinal cord lesions with nodular or granular aspects have also been detected in $S$. mansoni infections in humans (Nobre et al 2001). Isointensity on T1-weighted images and hyperintensity on T2-weighted images, associated with acute haemorrhagic spinal cord lesions, have been described in dogs infected with $A$. vasorum and Schistosoma (Nobre et al 2001, Wessmann et al 2006). Spinal cord enlargement on T1-weighted and T2-weighted images is a common finding in dogs and humans with nematode-associated myelopathy (Kanpittaya et al 2000, Jabbour et al 2011).

A definitive diagnosis of feline gurltiosis can only be made by post-mortem examination, demonstrating the presence of nematodes in the spinal cord vasculature (Gómez et al 2010, Guerrero et al 2011). A clinical history of chronic paraparesis or paraplegia (including signs of symmetric or asymmetric pelvic limb ataxia, tail paralysis and faecal or urinary incontinence) in potentially 


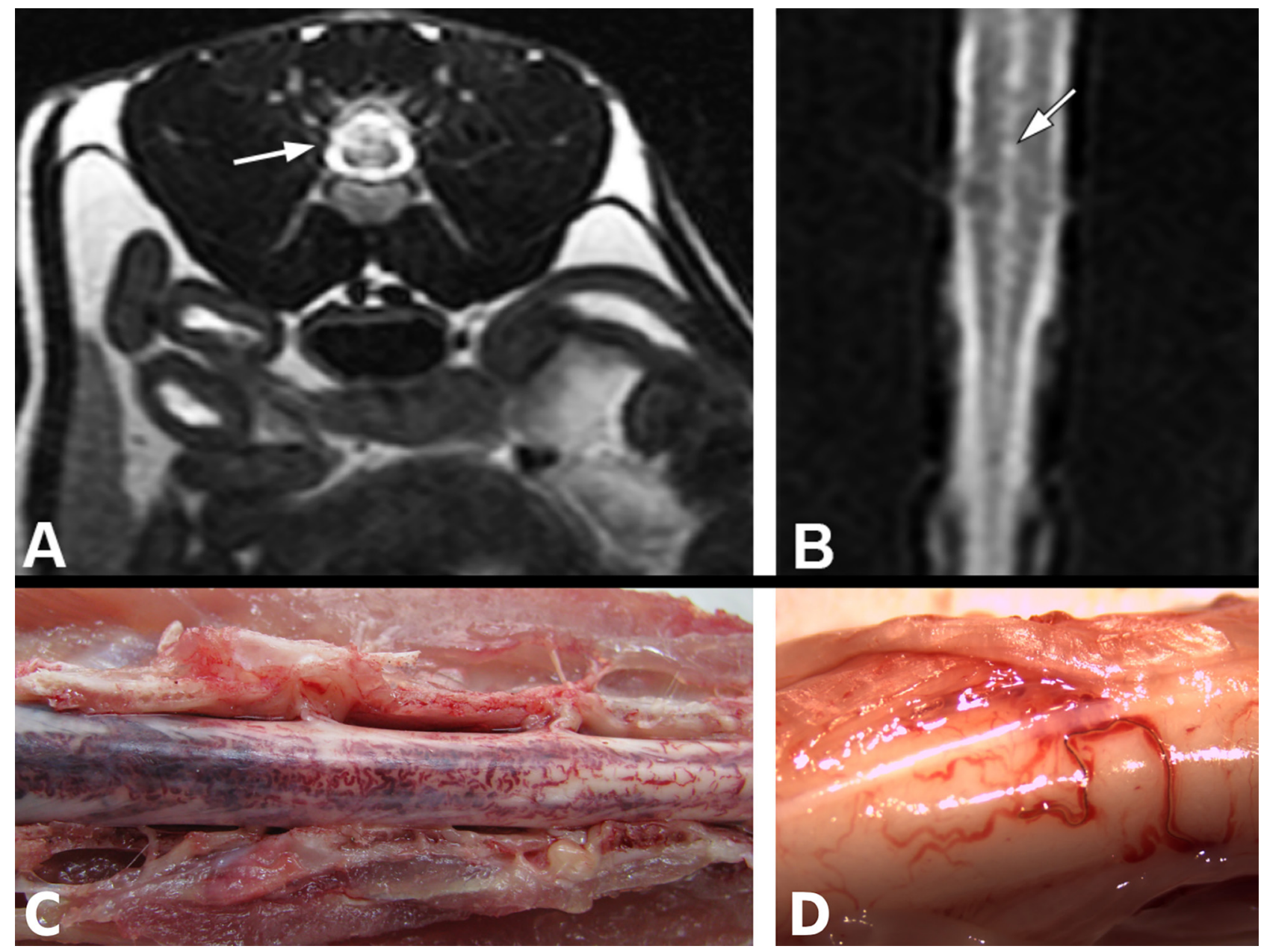

Figure 5. Magnetic resonance image of a cat with myelopathy due to Gurltia paralysans infection. Transverse T2W image showing hyperintense area in the spinal cord at level 3 (arrow) (A). Dorsal T2W image of a cat with vascular venous congestion and venous stasis of the dorsal spinal vein at the conus medullaris (arrow) (B). Macroscopic image of the lumbar spinal cord of a domestic cat with G. paralysans infection, demonstrating severe submeningeal vein congestion (C). Macroscopic image of the spinal cord of a domestic cat showing an adult nematode of G. paralysans inside a leptomeningeal vein (D).

endemic areas, laboratory findings (cerebrospinal fluid [CSF], haemography, faecal examination), and imaging findings are necessary to exclude other myelopathies and to establish a presumptive diagnosis of feline gurltiosis (Mieres et al 2013).

A cross-reactivity of G. paralysans and A. vasorum using a commercial serological test developed for the diagnosis of canine angiostrongylosis in domestic dogs has been recently evaluated for the use in domestic cats with gurltiosis. Preliminary results showed that the Angio Detect Test ${ }^{\mathrm{TM}}$ (IDEXX Laboratories), a rapid test designed to detect circulating antigen based on A. vasorum-specific antibodies, can be used as an effective test for cats displaying clinical signs of G. paralysans infection (Gómez et al 2020).

Novel molecular techniques for G. paralysans have been recently developed that could facilitate the clinical diagnosis of this nematode in infected animals (see below).

\section{MOLECULAR CHARACTERISATION OF G. PARALYSANS}

Members of the genus Gurltia are morphologically similar to those of the closely related genera of the Family Angiostrongylidae (Moroni et al 2012). Using sequences of the 28S rRNA D2-D3 region, the complete internal transcribed spacer 1 (ITS1) and ITS2 of the 5.8S rRNA, and the partial 18S rRNA gene (Gómez et al 2013, Hermosilla et al 2013, Muñoz et al 2017), confirmed that G. paralysans is a member of the family Angiostrongylidae and are a distinct taxonomic genus in the superfamily Metastrongyloidea (Muñoz et al 2017). A phylogenetic analysis showed that G. paralysans is most closely related to A. vasorum and Filaroides martis (figure 6).

Targeting a $717 \mathrm{bp}$ conserved nucleotide sequences of the D2-D3 region of the 28S rRNA gene, a semi-nested PCR method to detect $G$. paralysans and differentiate from these other nematodes has been developed (Hermosilla 


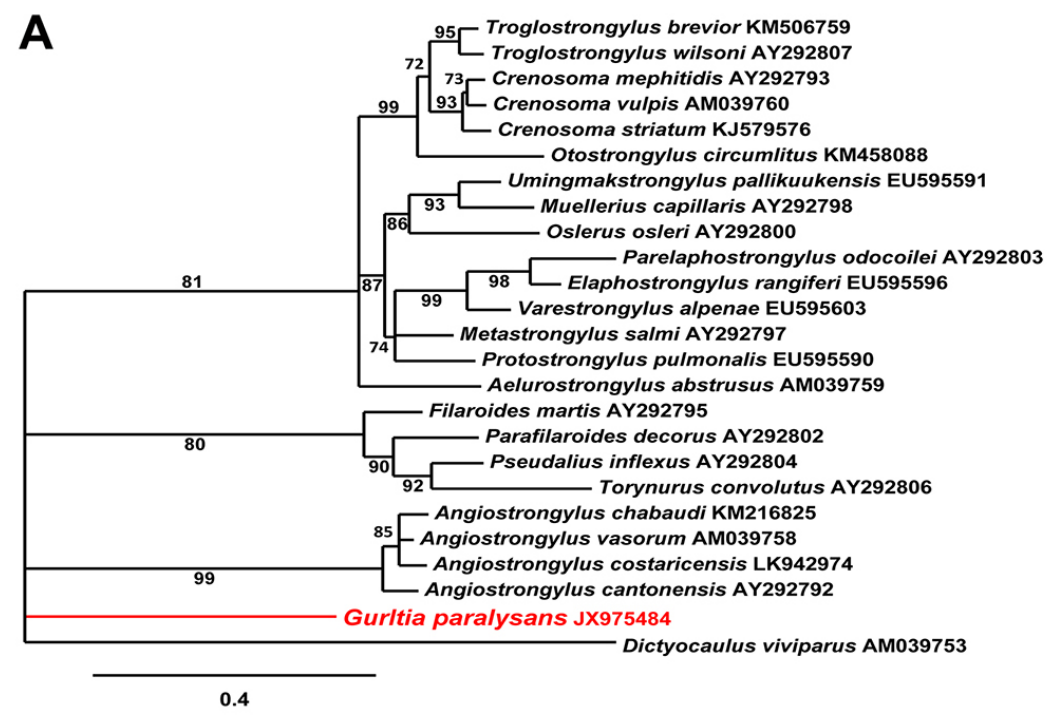

B

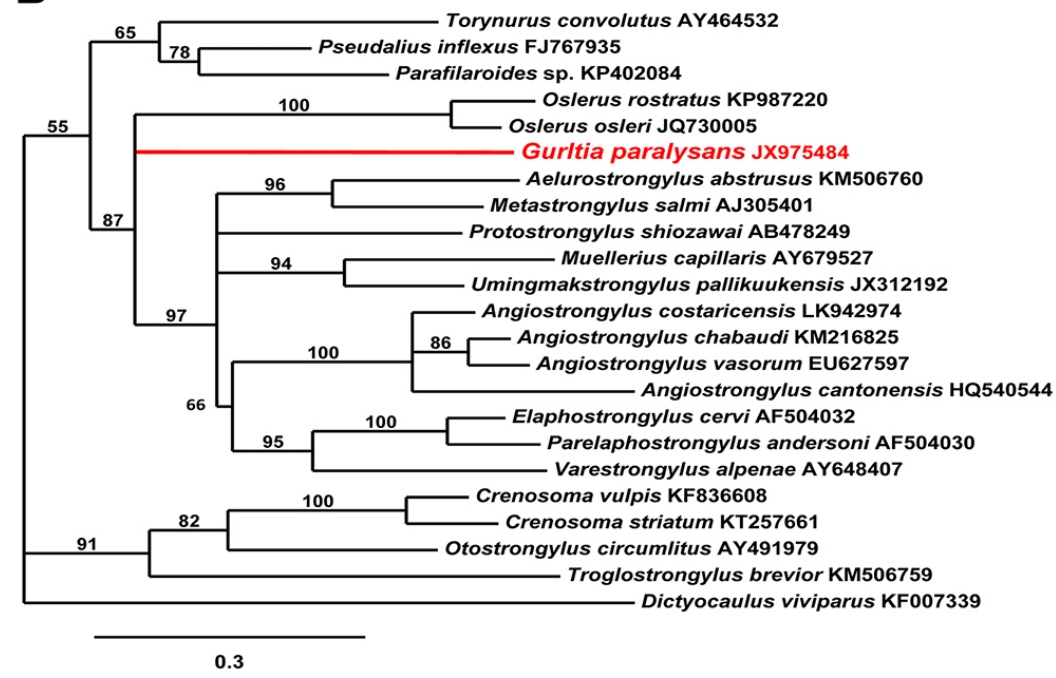

Figure 6. Phylogenetic relationships of Gurltia paralysans (red) and other metastrongyloid nematodes. Phylogenetic analyses were based on 28S rRNA D2-D3 (A) and ITS rDNA sequences (B) (taken from Muñoz et al 2017).

et al 2013, Muñoz et al 2017) (figure 7). The D2-D3 region of the parasite can be used as a molecular marker, and that this PCR is an effective diagnostic method for the identification of infected cats using serum and blood samples (Hermosilla et al 2013, Muñoz et al 2017). This PCR method also allows the detection of the larval stages of $G$. paralysans in potential $\mathrm{IH}$, and can be used in epidemiological prevalence studies in domestic cats. Using CSF samples, DNA fragments of $G$. paralysans has been successfully detected in 4 out of 6 CSF samples (figure 7) (López et al 2020).

\section{TREATMENT, PREVENTION, AND CONTROL}

The administration of four doses of ivermectin (0.2-0.4 $\mathrm{mg} / \mathrm{kg}$ ) at 1-weekly intervals have satisfactory outcomes in mild or moderate cases (Gómez et al 2012). Ricobendazole $(20 \mathrm{mg} / \mathrm{kg}$ ) and ivermectin has been also used combined in four adult cats with suspected feline gurltiosis (chronic ambulatory paraparesis) in an endemic area in Argentina (Guerrero et al 2011). The cats were observed for 5 weeks after their treatment to assess their clinical evolution. The preliminary results indicated no progression of the clinical condition, suggesting that some antiparasitic effect was achieved. However, no randomised trials of antihelminthics have been conducted for feline gurltiosis. Fenbendazole, milbemycin oxime, or moxidectin combined with imidacloprid may reduce the risk of $G$. paralysans infection, as they do for other related nematode species, such as $A$. vasorum, but further research is required.

No prepatent period has been observed for G. paralysans in vivo, and the monthly administration of drugs 


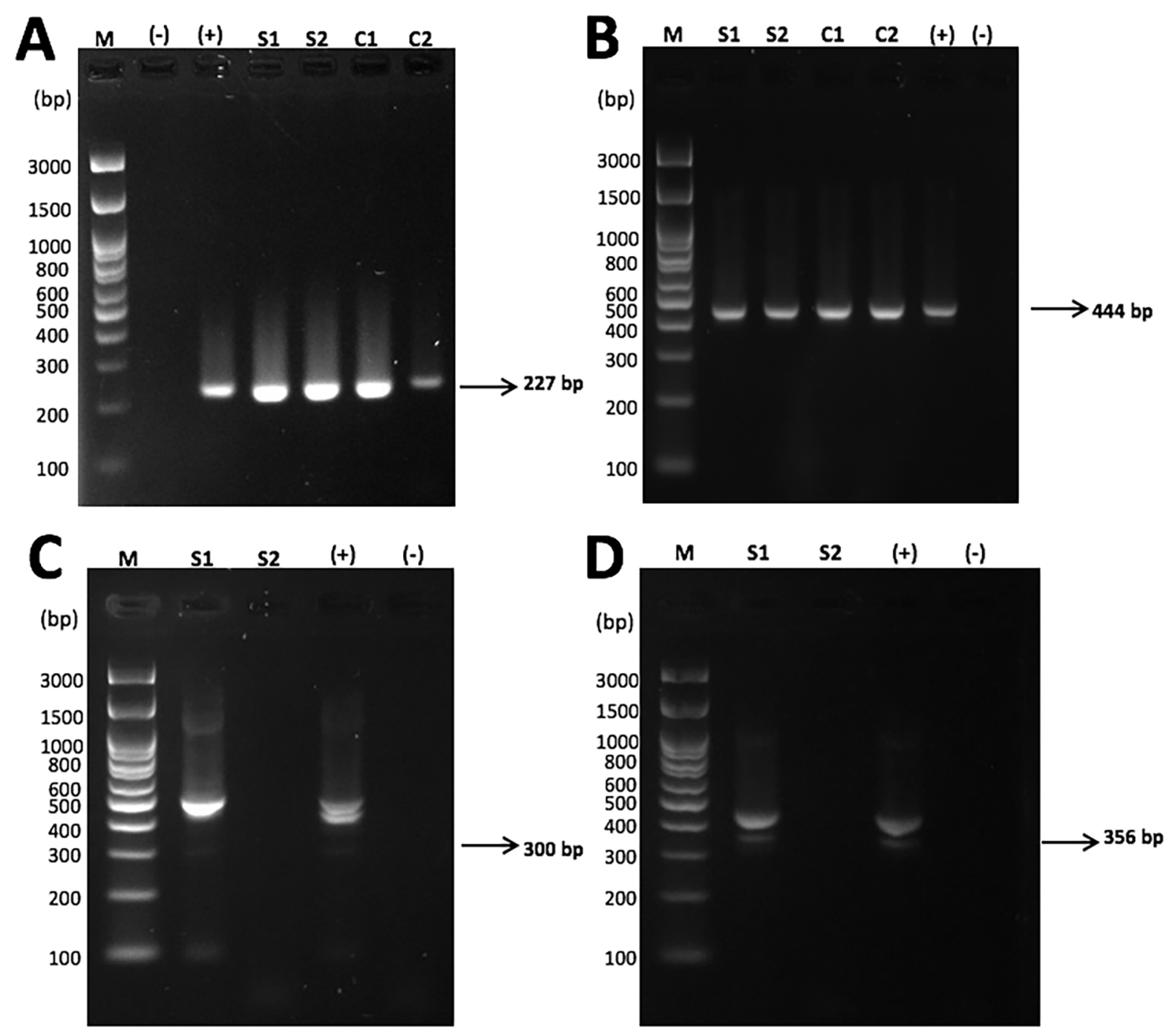

Figure 7. Molecular detection of Gurltia paralysans in the serum and cerebrospinal fluid of infected and uninfected domestic cats. A) Amplification of IRBP gene as the internal control in infected and uninfected domestic cats. PCR amplified a 227-bp fragment. B) Amplification of a sequence conserved in Metastrongylidae in serum and cerebrospinal fluid samples from infected and uninfected domestics cats, using the AaGp28Ss1 and AaGp28Sa1 universal primers. PCR product of $444 \mathrm{bp}$. C) Semi-nested Aelurostrongylus abstrusus PCR using specific AaGp28Sa1/Gp28Sa3 primers. PCR product of $300 \mathrm{bp}$. D) Semi-nested G. paralysans PCR using specific AaGp28Ss1/Gp28Sa3 primers. PCR product of $356 \mathrm{bp} . \mathrm{C} 1$ and $\mathrm{C} 2$, cerebrospinal fluid of domestic cats. In this case, only serum samples are shown in C and D. S1, infected cat. S2, uninfected cat. (+) the positive control and (-) the negative control. M, molecular size marker (100 bp ladder). The PCR products were run on a $2 \%$ agarose gel. Animals also showed the presence of G. paralysans specimen upon post-mortem examination.

containing macrocyclic lactone (i.e. ivermectin, selamectin, or milbemycin) might be considered a suitable prophylactic treatment for the prevention of $G$. paralysans infection in known endemic areas. The prevention of the infection by limiting access to $\mathrm{PH}$ or $\mathrm{IH}$ by maintaining cats indoor should be considered in endemic areas.

\section{CONCLUSIONS}

Gurltia paralysans is a metastrongyloid parasite that may cause chronic meningomyelitis in domestic cats in
South America. The geographic distribution of G. paralysans includes rural and peri-urban areas. The life cycle of the parasite is still unknown, but is probably indirect, as in other metastrongyloid nematodes. Further research is required to clarify the infection of $\mathrm{IH}$ and the transmission to $\mathrm{PH}$. The clinical signs of G. paralysans infection include progressive pelvic limb ataxia, paraparesis, paraplegia, faecal or urinary incontinence, and/or tail paralysis. The definitive in vivo diagnosis remains challenging, but the disease could be tentatively identified by clinical signs, haemography, CSF analysis, and imaging studies (CT and 
MRI). The PCR is currently a complementary diagnostic method, using serum and/or CSF samples. The definitive diagnosis still remains post-mortem based on the presence of larvae in the spinal cord. Preliminary reports suggest that the administration of macrocyclic lactones drugs may be useful in treating mild cases. Veterinarians and owners should be warned of the environmental control of definitive and intermediate hosts as an effective approach to reducing the likelihood of infection.

\section{REFERENCES}

Bono MF, Orcellet V, Marengo R, Bosio A, Junkers E, et al. 2016. A description of three cases of parasitic meningomyelitis in felines from the province of Santa Fé, Argentina. Parasitaria 74, 1-4.

Bourque A. 2002. Angiostrongylus vasorum infection in 2 dogs from Newfoundland. Can Vet J 43, 876.

Bowman DD, Hendrix CM, Lindsay DS, Barr SC. 2002. Metastrongyloidea. In: Feline Clinical Parasitology. $1^{\text {st }}$ ed. Iowa State University Press, Ames, Iowa, USA, Pp 272-273.

Cook S, Priestnall SL, Blake D, Meeson RL. 2015. Angiostrongylus vasorum causing severe granulomatous hepatitis with concurrent multiple acquired PSS. J Am Anim Hosp Assoc 51, 320-324.

Chai O, Shelef I, Brenner O, Dogadkin O, Aroch I, et al. 2008 Magnetic resonance imaging findings of spinal intramedullary spirocercosis. Vet Radiol Ultrasound 5, 456-459.

Dazzi CC, Santos A, Machado TP, Ataíde MW, Rodriguez R, et al. 2020. First case report of nematode parasitic myelopathy in a wild feline in Brazil. Braz J Vet Parasitol 29, e014619.

Figueroa N. 2017. Descripción histopatológica de lesiones encefálicas en gatos domésticos infectados con Gurltia paralysans. DVM Thesis, Universidad Austral De Chile, Valdivia, Chile

Foronda P, López-González M, Miquel J, Torres J, Segovia M, et al. 2010. Finding of Parastrongylus cantonensis (Chen, 1935) in Rattus rattus in Tenerife, Canary Islands (Spain). Acta Trop 114, 123-127.

Gómez M, Freeman, L. 2003. Revisión del plexo venoso vertebral en el perro. Int J Morphol 21, 237-244.

Gómez M, Mieres M, Moroni M, Mora A, Barrios N, et al. 2010. Meningomyelitis due to nematode infection in four cats. Vet Parasitol 170, 327-330.

Gómez M, Rojas M, Mieres M, Moroni M, Muñoz P. 2011. Clinical, clinicopathological and pathological findings in 7 domestic cats with paraparesis/plegia produced by nematodes in southern Chile. Proceedings $24^{\text {th }}$ Symposium ESVN-ECVN, Trier, Germany.

Gómez G, Aranzazu D, Alzate A, Chaparro JJ. 2011. Domestic cat paraplegia compatible with Gurltia paralysans nematode. First cases reported in Colombia. Rev Colomb Cienc Pecu 24, 663-669.

Gómez M, Mieres M, Lillo C, Rojas M, Muñoz M, et al. 2012. Clinical and imaging findings in feline myelopathy induced by Gurltia paralysans: a neglected neuroparasite in domestic cats. DVG Parasitologie Meeting, Hannover, Germany.

Gómez M, García C, Maldonado I, Pantchev N, Taubert A, et al. 2020. Intra Vitam diagnosis of neglected Gurltia paralysans infections in domestic cats (Felis catus) by a commercial serology test for canine angiostrongylosis and insights into clinical and histopathological findings - four-case report. Pathogens 9, 921.

Gredal H, Willesen JL, Jensen HE, Nielsen O, Kristensn A, et al. 2011. Acute neurological signs as the predominant clinical manifestation in four dogs with Angiostrongylus vasorum infections in Denmark. Acta Vet Scand 53, 43-50.

Guerrero I, Paludi A, Saumell L. 2011. Primera descripción en Argentina de Gurltia paralysans en un felino doméstico. DVM Thesis, Universidad Del Centro De La Provincia Buenos Aires, Tandil, Argentina.

Hermosilla C, Gómez M, Muñoz P, Moroni M, Mieres M, et al. 2013. Molekulare Charakterisierung von Gurltia paralysans und
Etablierung einer Speziesspezifischen PCR. DVG Parasitologie Meeting, Giessen, Germany.

Jabbour R, Kanj S, Sawaya R, Awar G, Houranim, et al. 2011. Toxocara canis myelitis: clinical features, magnetic resonance imaging (MRI) findings, and treatment outcome in 17 patients. Medicine 90, 337-343.

Jara C. 2018. Caracterización de la respuesta leucocitaria y glial en gatos con meningomielitis por Gurltia paralysans. DVM Thesis, Universidad Austral De Chile, Valdivia, Chile.

Kanpittaya J, Jitpimolmard S, Tiamkao S, Mairiange E. 2000. MR findings of eosinophilic meningoencephalitis attributed to Angiostrongylus cantonensis. AJNR Am J Neuroradiol 21, 1090-1094.

Katchanov J, Nawa Y. 2010. Helminthic invasion of the central nervous system: Many roads lead to Rome. Parasitol Int 59, 491-496.

Lange MK, Penagos-Tabares F, Vélez J, Gutiérrez J, Hirzmann J, et al. 2018. Regional report on Angiostrongylus vasorum in Colombia: Genetic similarity to European lineage. Vet Parasitol Reg Stud Reports 13, 21-23.

Levine ND. 1968. Nematode Parasites of Domestic Animals and of Man. Burgess Publishing Co., Minneapolis, Minn., USA. Pp 291.

López-Contreras F, Rojas-Barón L, Gómez M, Morera F, Sepúlveda P, et al. 2020. Molecular detection of Gurltia paralysans by semi-nested PCR in cerebrospinal fluid and serum samples from domestic cats (Felis catus). Animals 10, 1169.

Maksimov P, Hermosilla C, Taubert A, Staubach C, Sauter-Louis C, et al. 2017. GIS-supported epidemiological analysis on canine Angiostrongylus vasorum and Crenosoma vulpis infections in Germany. Parasit Vectors 28, 108.

Melo Neto GL, Silva R, Alves RC, Gomes R, Medeiros A, et al. 2019. Infecção por Gurltia paralysans em gatos domésticos no Eestado de Pernambuco, Brasil. Acta Scientiae Veterinariae 47, 418.

Mieres M, Gómez M, Lillo C, Rojas M, Moroni M, et al. 2013. Clinical, imaging, and pathologic characteristics of Gurltia paralysans myelopathy in domestic cats from Chile. Vet Radiol Ultrasound 54, 237-244.

Morgan ER, Jefferies R, Krajewski M, Ward P, Shaw, SE. 2009 Canine pulmonary angiostrongylosis: the influence of climate on parasite distribution. Parasitol Int 58, 406-410.

Moroni M, Muñoz P, Gómez M, Mieres M, Rojas M, et al. 2012. Gurltia paralysans (Wolffhügel, 1933): description of adults and additional case reports of neurological diseases in three domestic cats from southern Chile. Vet Parasitol 184, 377-380.

Moroni M, Muñoz P, Mieres M, Gómez M, Vera F. 2016. Severe myelopathy with thrombophlebitis caused by Gurltia paralysans infection in a cat. Vet Rec Case Rep 4, e000327.

Muñoz P, Hirzmann J, Rodriguez E, Moroni M, Taubert A, et al. 2017. Redescription and first molecular characterization of the little known feline neurotropic nematode Gurltia paralysans (Nematoda: Metastrongyloidea). Vet Parasitol Reg Stud Reports 10, 119-125.

Nobre V, Silva L, Ribas J, et al. 2001. Schistosomal myeloradiculopathy due to Schistosoma mansoni: Report on 23 cases. Mem Inst Oswaldo Cruz 96, 137-141.

Oliveira B. 2015. Politraumatismo em gato-maracajá (Leopardus wieddi) com infecção por Gurltia paralysans. Integrando prácticas e transvesalizando saberes. Semana do Conhecimiento UPF, Passo Fundo, Brazil.

Paz J, Valente M, Casella EB, Marques-Dias MJ. 2002. Spinal cord schistosomiasis in children: analysis of seven cases. Arq Neuropisiquiatr 60, 224-230.

Peña G. 2014. Hallazgos clínicos, hematológicos, bioquímicos y de lavado broncoalveolar en 8 gatos domésticos (Felis catus) con paraparesis/ plejia producida por Gurltia paralysans. DVM Thesis, Universidad Austral De Chile, Valdivia.

Read AF, Sharping A. 1995. The evolution of tissue migration by parasitic nematode larvae. Parasitology 111, 359-371.

Rinaldi L, Cortese L, Meomartino L, Pagano T, Pepe P, et al. 2014. Angiostrongylus vasorum: epidemiological, clinical and histopathological insights. BMC Vet Res 10, 236.

Rivero R, Matto C, Adrien M, Nan F, Bell T, et al. 2011. Parasite meningomyelitis in cats in Uruguay. Rev Bras Parasitol Vet 20, 259-261. 
Rodríguez E. 2013. Descripción morfológica del nematodo Gurltia paralysans. DVM Thesis, Universidad Austral De Chile, Valdivia, Chile.

Rojas M. 2011. Análisis clínico-patológico en 7 gatos domésticos (Felis catus) con paraparesis/plejia producida por Gurltia paralysans. DVM Thesis, Universidad Austral De Chile, Valdivia.

Sepúlveda P. 2018. Detección de estados larvales de Gurltia paralysans (Wolffhügel, 1933), mediante digestión enzimática y PCR en gastrópodos terrestres de la localidad de Punucapa, Valdivia, Chile. MSc Dissertation. Universidad Austral de Chile, Valdivia, Chile.

Shahlaie K, Hawk MW, Hu BR, Theis JH, Kim KD. 2005. Parasitic central nervous system infections: Echinococcus and Schistosoma. Rev Neurol Dis 76-85.

Togni M, Panziera W, Souza T, Oliveira JC, Mazzanti A, et al. 2013. Aspectos epidemiológicos, clínicos e anatomopatologicos da infecção por Gurltia paralysans em gatos. Pesq Vet Bras 33, 363-371.

Traversa D, Di Cesare A, Conboy G. 2010. Canine and feline cardiopulmonary parasitic nematodes in Europe: Emerging and underestimated. Parasit Vectors 3, 62.
Udiz-Rodríguez R, Garcia-Livia K, Valladares-Salmerón M, DortaAlmenar MN, Martín-Carrillo N, et al. 2018. First ocular report of Gurltia paralysans (Wolffhügel, 1933) in cat. Vet Parasitol 15, 74-77.

Verscheure A. 2014. Hallazgos histológicos en hígado, pulmón y riñón de gatos domésticos con paraparesia o paraplejia por Gurltia paralysans. DVM Thesis, Universidad Austral De Chile, Valdivia.

Vienenkötter J, Hermosilla C, Taubert A, Herden C, Gómez M, et al. 2015. Spinal cord lesions in a cat infected with Gurltia paralysans. J Comp Pathol 152, 80.

Wessmann A, Lu D, Lamb CR, Smyth B, Mantis P, et al. 2006. Brain and spinal cord haemorrhages associated with Angiostrongylus vasorum infection in four dogs. Vet Rec 158, 858-863.

Wolffhügel K. 1933. Paraplegia cruralis parasitaria felis causada por Gurltia paralysans nov. gen., n. sp. Rev Chil Hist Nat 190-192.

Wolffhügel K. 1934. Paraplegia cruralis parasitaria felis durch Gurltia paralysans nov. gen., nov. sp. (Nematoda). 2. Infektkr Haustiere $46,28-47$. 
